\title{
A SHARP VERSION OF THE FORELLI-RUDIN TYPE ESTIMATES ON THE UNIT REAL BALL
}

\author{
Bingwen Lin, Congwen Liu and Jun Wang \\ University of Science and Technology of China, School of Mathematical Sciences \\ Hefei, Anhui 230026, P. R. China; linbw@mail.ustc.edu.cn \\ Chinese Academy of Sciences, USTC, Wu Wen-Tsun Key Laboratory of Mathematics \\ and University of Science and Technology of China, School of Mathematical Sciences \\ Hefei, Anhui 230026, P. R. China; cwliu@ustc.edu.cn \\ University of Science and Technology of China, School of Mathematical Sciences \\ Hefei, Anhui 230026, P. R. China; onedream@mail.ustc.edu.cn
}

\begin{abstract}
The purpose of this short note is to establish a sharp version of Forelli-Rudin type estimates for certain integrals on the real ball.
\end{abstract}

\section{Introduction}

Let $\mathbf{B}_{n}$ denote the unit ball in $\mathbf{R}^{n}$ and $\mathbf{S}^{n-1}$ its boundary. By $d V$ we denote the Lebesgue measure on $\mathbf{R}^{n}$, normalized so that $V\left(\mathbf{B}_{n}\right)=1$, and $d \sigma$ the surface measure on $\mathbf{S}^{n-1}$ so that $\sigma\left(\mathbf{S}^{n-1}\right)=1$. For $x \in \mathbf{B}_{n}, s \in \mathbf{R}$ and $t>-1$, define

$$
\mathbf{I}_{s}(x):=\int_{\mathbf{S}^{n-1}} \frac{d \sigma(\zeta)}{|x-\zeta|^{n-1+s}}
$$

and

$$
\mathbf{J}_{s, t}(x):=\int_{\mathbf{B}_{n}} \frac{\left(1-|y|^{2}\right)^{t} d V(y)}{\llbracket x, y \rrbracket^{n+t+s}}
$$

where $\llbracket x, y \rrbracket$ denotes the Ahlfors bracket

$$
\llbracket x, y \rrbracket:=\left(1-2 x \cdot y+|x|^{2}|y|^{2}\right)^{1 / 2} .
$$

The following result is well-known to experts in complex analysis and harmonic analysis. See, for instantce, [7, Lemmas 2.9 and 2.10], [5, Proposition 2.1] or [11, lemma 4.4].

Theorem A. Let $\mathbf{I}_{s}(x)$ and $\mathbf{J}_{s, t}(x)$ be defined as in (1.1) and (1.2). Then

$$
I_{s}(x) \approx \mathbf{J}_{s, t}(x) \approx \begin{cases}1, & \text { if } s<0 \\ \log \frac{1}{1-|x|^{2}}, & \text { if } s=0 \\ \left(1-|x|^{2}\right)^{-s}, & \text { if } s>0\end{cases}
$$

as $|x| \rightarrow 1^{-}$, where the notation $a(x) \approx b(x)$ means that the ratio $a(x) / b(x)$ is bounded from above and below by two positive constants as $|x| \rightarrow 1^{-}$.

doi:10.5186/aasfm.2016.4114

2010 Mathematics Subject Classification: Primary 26D15, 30H20; Secondary 33C05, 33C90.

Key words: Forelli-Rudin type estimates, integral operators, norm estimates, harmonic Bergman projection.

This work was supported by the National Natural Science Foundation of China grant 11171318, 11471301 and by OATF, USTC. 
In view of the analogy with the classical Forelli-Rudin estimates (see, e.g. [12, Proposition 1.4.10]), we shall call the above result the Forelli-Rudin type estimates on the real ball. The purpose of this note is to present a sharp version of these estimates, which can also be viewed as an analogue of [8, Theorem 1.3] in the setting of the unit real ball. More precisely, our main results are as follows.

Theorem 1.1. Let $s \in \mathbf{R}$ and let $\mathbf{I}_{s}(x)$ be defined as in (1.1).

(i) If $-1 \leq s<0$ or $s \leq 1-n$, then for all $x \in \mathbf{B}_{n}$,

$$
1 \leq \mathbf{I}_{s}(x) \leq \frac{\Gamma\left(\frac{n}{2}\right) \Gamma(-s)}{\Gamma\left(\frac{1-s}{2}\right) \Gamma\left(\frac{n-1-s}{2}\right)} .
$$

(ii) If $1-n<s<-1$, then for all $x \in \mathbf{B}_{n}$,

$$
\frac{\Gamma\left(\frac{n}{2}\right) \Gamma(-s)}{\Gamma\left(\frac{1-s}{2}\right) \Gamma\left(\frac{n-1-s}{2}\right)} \leq \mathbf{I}_{s}(x) \leq 1
$$

(iii) If $0<s \leq 1$ or $s \geq n-1$, then for all $x \in \mathbf{B}_{n}$,

$$
1 \leq\left(1-|x|^{2}\right)^{s} \mathbf{I}_{s}(x) \leq \frac{\Gamma\left(\frac{n}{2}\right) \Gamma(s)}{\Gamma\left(\frac{1+s}{2}\right) \Gamma\left(\frac{n-1+s}{2}\right)} .
$$

(iv) If $1<s<n-1$, then for all $x \in \mathbf{B}_{n}$,

$$
\frac{\Gamma\left(\frac{n}{2}\right) \Gamma(s)}{\Gamma\left(\frac{1+s}{2}\right) \Gamma\left(\frac{n-1+s}{2}\right)} \leq\left(1-|x|^{2}\right)^{s} \mathbf{I}_{s}(x) \leq 1 \text {. }
$$

(v) If $s=0$ and $n=2$, then for all $x \in \mathbf{B}_{n}$,

$$
\frac{1}{\pi} \leq|x|^{2}\left(\log \frac{1}{1-|x|^{2}}\right)^{-1} \mathbf{I}_{0}(x) \leq 1 .
$$

(vi) If $s=0$ and $n \geq 3$, then for all $x \in \mathbf{B}_{n}$,

$$
\frac{1}{2} \leq|x|\left(\log \frac{1+|x|}{1-|x|}\right)^{-1} \quad \mathbf{I}_{0}(x) \leq \frac{\Gamma\left(\frac{n}{2}\right)}{\sqrt{\pi} \Gamma\left(\frac{n-1}{2}\right)} .
$$

Furthermore, all these inequalities are sharp.

Theorem 1.2. Let $s \in \mathbf{R}, t>-1$ and let $\mathbf{J}_{s, t}(x)$ be defined as in (1.2).

(i) If $-2-t \leq s<0$ or $s \leq-n-t$, then for all $x \in \mathbf{B}_{n}$,

$$
\frac{\Gamma\left(\frac{n}{2}+1\right) \Gamma(1+t)}{\Gamma\left(\frac{n}{2}+1+t\right)} \leq \mathbf{J}_{s, t}(x) \leq \frac{\Gamma\left(\frac{n}{2}+1\right) \Gamma(1+t) \Gamma(-s)}{\Gamma\left(\frac{2+t-s}{2}\right) \Gamma\left(\frac{n+t-s}{2}\right)} .
$$

(ii) If $-n-t<s<-2-t$, then for all $x \in \mathbf{B}_{n}$,

$$
\frac{\Gamma\left(\frac{n}{2}+1\right) \Gamma(1+t) \Gamma(-s)}{\Gamma\left(\frac{2+t-s}{2}\right) \Gamma\left(\frac{n+t-s}{2}\right)} \leq \mathbf{J}_{s, t}(x) \leq \frac{\Gamma\left(\frac{n}{2}+1\right) \Gamma(1+t)}{\Gamma\left(\frac{n}{2}+1+t\right)} .
$$

(iii) If $0<s \leq 2+t$ or $s \geq n+t$, then for all $x \in \mathbf{B}_{n}$,

$$
\frac{\Gamma\left(\frac{n}{2}+1\right) \Gamma(1+t)}{\Gamma\left(\frac{n}{2}+1+t\right)} \leq\left(1-|x|^{2}\right)^{s} \mathbf{J}_{s, t}(x) \leq \frac{\Gamma\left(\frac{n}{2}+1\right) \Gamma(1+t) \Gamma(s)}{\Gamma\left(\frac{2+t+s}{2}\right) \Gamma\left(\frac{n+t+s}{2}\right)} .
$$

(iv) If $2+t<s<n+t$, then for all $x \in \mathbf{B}_{n}$,

$$
\frac{\Gamma\left(\frac{n}{2}+1\right) \Gamma(1+t) \Gamma(s)}{\Gamma\left(\frac{2+t+s}{2}\right) \Gamma\left(\frac{n+t+s}{2}\right)} \leq\left(1-|x|^{2}\right)^{s} \mathbf{J}_{s, t}(x) \leq \frac{\Gamma\left(\frac{n}{2}+1\right) \Gamma(1+t)}{\Gamma\left(\frac{n}{2}+1+t\right)} .
$$


(v) If $-1<t \leq 0$ and $n=2$, then for all $x \in \mathbf{B}_{n}$,

$$
\frac{\Gamma(1+t)}{\Gamma^{2}\left(\frac{2+t}{2}\right)} \leq|x|^{2}\left(\log \frac{1}{1-|x|^{2}}\right)^{-1} \mathbf{J}_{0, t}(x) \leq \frac{1}{1+t} .
$$

(vi) If either $t>-1$ and $n \geq 3$, or $t \geq 0$ and $n \geq 2$, then for all $x \in \mathbf{B}_{n}$,

$$
\frac{\Gamma\left(\frac{n}{2}+1\right) \Gamma(1+t)}{2 \Gamma\left(\frac{n}{2}+1+t\right)} \leq|x|\left(\log \frac{1+|x|}{1-|x|}\right)^{-1} \mathbf{J}_{0, t}(x) \leq \frac{\Gamma\left(\frac{n}{2}+1\right) \Gamma(1+t)}{\Gamma\left(\frac{n+t}{2}\right) \Gamma\left(\frac{2+t}{2}\right)} .
$$

Furthermore, all these inequalities are sharp.

The following corollary is more useful in applications than Theorems 1.1 and 1.2.

Corollary 1.3. Let $s>0$ and $t>-1$. We have

$$
\begin{aligned}
& \sup _{x \in \mathbf{B}_{n}} \int_{\mathbf{S}^{n-1}} \frac{\left(1-|x|^{2}\right)^{s} d \sigma(\zeta)}{|x-\zeta|^{n-1+s}} \\
& = \begin{cases}\frac{\Gamma\left(\frac{n}{2}\right) \Gamma(s)}{\Gamma\left(\frac{1+s}{2}\right) \Gamma\left(\frac{n-1+s}{2}\right)}, & \text { if } 0<s \leq 1 \text { or } s \geq n-1 ; \\
1, & \text { if } 1<s<n-1,\end{cases}
\end{aligned}
$$

and

$$
\begin{aligned}
& \sup _{x \in \mathbf{B}_{n}} \int_{\mathbf{B}_{n}} \frac{\left(1-|x|^{2}\right)^{s}\left(1-|y|^{2}\right)^{t}}{\llbracket x, y \rrbracket^{n+t+s}} d V(y) \\
& = \begin{cases}\frac{\Gamma\left(\frac{n}{2}+1\right) \Gamma(1+t) \Gamma(s)}{\Gamma\left(\frac{2+t+s}{2}\right) \Gamma\left(\frac{n+t+s}{2}\right)}, & \text { if } 0<s \leq 2+t \text { or } s \geq n+t ; \\
\frac{\Gamma\left(\frac{n}{2}+1\right) \Gamma(1+t)}{\Gamma\left(\frac{n}{2}+1+t\right)}, & \text { if } 2+t<s<n+t .\end{cases}
\end{aligned}
$$

\section{Lemmas}

The Gaussian hypergeometric function is defined by

$$
{ }_{2} F_{1}\left[\begin{array}{c}
a, b \\
c
\end{array} ; \varrho\right]=\sum_{k=0}^{\infty} \frac{(a)_{k}(b)_{k}}{(c)_{k}} \frac{\varrho^{k}}{k !}
$$

for $\varrho \in(-1,1)$, with $c \neq 0,-1,-2, \cdots$. Here $(a)_{k}$ denotes the shifted factorial

$$
\begin{aligned}
& (a)_{0}=1, \\
& (a)_{k}=a(a+1) \cdots(a+k-1), \quad k=1,2, \ldots .
\end{aligned}
$$

We should mention that this function is usually defined for complex parameters and argument. However, the above definition is enough for our purpose in this paper.

Lemma 2.1. Suppose that $a, b \in \mathbf{R}$ and $c>\max \{a, b, a+b,-1\}$. If $a b c \geq 0$ then the function $\varrho \mapsto{ }_{2} F_{1}\left[\begin{array}{c}a, b \\ c\end{array} ; \varrho\right]$ is increasing from $[0,1)$ onto $[1, B)$, otherwise it is decreasing from $[0,1)$ onto $(B, 1]$. Here

$$
B:=\frac{\Gamma(c) \Gamma(c-a-b)}{\Gamma(c-a) \Gamma(c-b)} .
$$


Proof. Combining the differentiation formula (see [3, p. 94 (2.5.1)])

$$
\frac{d}{d \varrho}{ }_{2} F_{1}\left[\begin{array}{c}
a, b \\
c
\end{array} ; \varrho\right]=\frac{a b}{c}{ }_{2} F_{1}\left[\begin{array}{c}
a+1, b+1 \\
c+1
\end{array} ; \varrho\right]
$$

and the Euler transformation formula (see [3, Theorem 2.2.5])

$$
{ }_{2} F_{1}\left[\begin{array}{c}
a, b \\
c
\end{array} ; \varrho\right]=(1-\varrho)^{c-a-b}{ }_{2} F_{1}\left[\begin{array}{c}
c-a, c-b \\
c
\end{array}\right]
$$

we obtain

$$
\frac{d}{d \varrho}{ }_{2} F_{1}\left[\begin{array}{c}
a, b \\
c
\end{array} ; \varrho\right]=\frac{a b}{c}(1-\varrho)^{c-a-b-1}{ }_{2} F_{1}\left[\begin{array}{c}
c-a, c-b \\
c+1
\end{array} \text { + }\right] .
$$

Note that the assumption $c>\max \{a, b, a+b,-1\}$ implies that the last hypergeometric function is nonnegative on $[0,1)$, since its Taylor coefficients are all positive. Hence $\frac{d}{d \varrho}{ }_{2} F_{1}\left[\begin{array}{c}a, b \\ c\end{array} ; \varrho\right] \geq 0$ on $[0,1)$ if and only if $a b c \geq 0$.

The limiting value as $\varrho \rightarrow 0$ follows from the series expansion, while the one as $\varrho \rightarrow 1^{-}$follows from the Gauss relation (see [3, Theorem 2.2.2])

$$
{ }_{2} F_{1}\left[\begin{array}{c}
a, b \\
c
\end{array} ; 1^{-}\right]=\frac{\Gamma(c) \Gamma(c-a-b)}{\Gamma(c-a) \Gamma(c-b)} .
$$

The lemma is proved.

Recall that ${ }_{2} F_{1}\left[\begin{array}{c}a, b \\ c\end{array} ; \varrho\right]$ is called zero-balanced if $c=a+b$. We shall need the following result for the zero-balanced hypergeometric functions from [1].

Lemma 2.2. [1, Theorem 1.4] If $0<a, b<1$, then the function

$$
\varrho \mapsto \varrho\left(\log \frac{1}{1-\varrho}\right)^{-1}{ }_{2} F_{1}\left[\begin{array}{c}
a, b \\
a+b
\end{array} \varrho\right]
$$

is decreasing on $(0,1)$, and for all $\varrho \in(0,1)$, we have

$$
\frac{\Gamma(a+b)}{\Gamma(a) \Gamma(b)} \frac{1}{\varrho} \log \frac{1}{1-\varrho} \leq{ }_{2} F_{1}\left[\begin{array}{c}
a, b \\
a+b
\end{array} ; \varrho\right] \leq \frac{1}{\varrho} \log \frac{1}{1-\varrho} .
$$

Moreover, the inequalities in (2.5) are sharp.

The following proposition appears to be new and of independent interest.

Proposition 2.3. If $a, b>0$ and $a b>\max \{1 / 2,(a+b) / 3\}$, then the function

$$
\varrho \mapsto \sqrt{\varrho}\left(\log \frac{1+\sqrt{\varrho}}{1-\sqrt{\varrho}}\right)^{-1}{ }_{2} F_{1}\left[\begin{array}{c}
a, b \\
a+b
\end{array} \varrho\right]
$$

is increasing on $(0,1)$, and for all $\varrho \in(0,1)$, we have

$$
\frac{1}{2 \sqrt{\varrho}} \log \frac{1+\sqrt{\varrho}}{1-\sqrt{\varrho}} \leq{ }_{2} F_{1}\left[\begin{array}{c}
a, b \\
a+b
\end{array} ; \varrho\right] \leq \frac{\Gamma(a+b)}{\Gamma(a) \Gamma(b)} \frac{1}{\sqrt{\varrho}} \log \frac{1+\sqrt{\varrho}}{1-\sqrt{\varrho}} .
$$

Moreover, the inequalities in (2.6) are sharp.

The proof follows the same lines as that of Lemma 2.2. We first recall the following elementary Lemma from [2]. 
Lemma 2.4. [2, Lemma 2.2] Let $\alpha, \beta \in \mathbf{R}$, and $\varphi, \psi:[\alpha, \beta] \rightarrow \mathbf{R}$ be continuous functions that are differentiable on $(\alpha, \beta)$, with $\varphi(\alpha)=\psi(\alpha)=0$ or $\varphi(\beta)=\psi(\beta)=0$. Assume that $\psi^{\prime}(x) \neq 0$ for all $x \in(\alpha, \beta)$. If $\varphi^{\prime} / \psi^{\prime}$ is increasing (decreasing) on $(\alpha, \beta)$, then so is $\varphi / \psi$.

Proof of Proposition 2.3. Set

$$
\varphi(\varrho):=\sqrt{\varrho}_{2} F_{1}\left[\begin{array}{c}
a, b \\
a+b
\end{array} ; \varrho\right] \quad \text { and } \quad \psi(\varrho):=\log \frac{1+\sqrt{\varrho}}{1-\sqrt{\varrho}}
$$

then $\varphi(0)=\psi(0)=0$ and

$$
\begin{aligned}
\frac{\varphi^{\prime}(\varrho)}{\psi^{\prime}(\varrho)}= & \sqrt{\varrho}(1-\varrho)\left(\frac{1}{2 \sqrt{\varrho}}{ }_{2} F_{1}\left[\begin{array}{c}
a, b \\
a+b
\end{array} \vdots \varrho\right]+\frac{a b}{a+b} \sqrt{\varrho}{ }_{2} F_{1}\left[\begin{array}{c}
a+1, b+1 \\
a+b+1
\end{array} ; \varrho\right]\right) \\
= & (1-\varrho)\left(\sum_{k=0}^{\infty} \frac{(a)_{k}(b)_{k}}{2(a+b)_{k}} \frac{\varrho^{k}}{k !}+\frac{a b}{a+b} \sum_{k=0}^{\infty} \frac{(a+1)_{k}(b+1)_{k}}{(a+b+1)_{k}} \frac{\varrho^{k+1}}{k !}\right) \\
= & \sum_{k=0}^{\infty} \frac{(a)_{k}(b)_{k}}{2(a+b)_{k}} \frac{\varrho^{k}}{k !}+\sum_{k=0}^{\infty}\left(\frac{(a)_{k+1}(b)_{k+1}}{(a+b)_{k+1}}-\frac{(a)_{k}(b)_{k}}{2(a+b)_{k}}\right) \frac{\varrho^{k+1}}{k !} \\
& -\sum_{k=0}^{\infty} \frac{(a)_{k+1}(b)_{k+1}}{(a+b)_{k+1}} \frac{\varrho^{k+2}}{k !}
\end{aligned}
$$

Hence

$$
\begin{aligned}
& 2 \frac{\varphi^{\prime}(\varrho)}{\psi^{\prime}(\varrho)}-1 \\
& =\frac{a b}{a+b} \varrho+\left(\frac{2 a b}{a+b}-1\right) \varrho+\sum_{k=0}^{\infty} \frac{(a)_{k+2}(b)_{k+2}}{(a+b)_{k+2}} \frac{\varrho^{k+2}}{(k+2) !} \\
& \quad+\sum_{k=0}^{\infty}\left(2 \frac{(a)_{k+2}(b)_{k+2}}{(a+b)_{k+2}}-\frac{(a)_{k+1}(b)_{k+1}}{(a+b)_{k+1}}\right) \frac{\varrho^{k+2}}{(k+1) !}-2 \sum_{k=0}^{\infty} \frac{(a)_{k+1}(b)_{k+1}}{(a+b)_{k+1}} \frac{\varrho^{k+2}}{k !} \\
& =\left(\frac{3 a b}{a+b}-1\right) \varrho+\sum_{k=0}^{\infty}[(k+1)(2 a b-1)+(3 a b-a-b)] \frac{(a)_{k+1}(b)_{k+1}}{(a+b)_{k+2}} \frac{\varrho^{k+2}}{(k+2) !}
\end{aligned}
$$

in which all the coefficients are positive. Thus $\varphi^{\prime} / \psi^{\prime}$ is increasing on $(0,1)$, thereby so is $\varphi / \psi$, by Lemma 2.4. The first assertion of the propostion is proved.

To prove the second assertion, it suffices to note that

$$
\lim _{\varrho \rightarrow 0^{+}} \frac{\varphi(\varrho)}{\psi(\varrho)}=\lim _{\varrho \rightarrow 0^{+}} \sqrt{\varrho}\left(\log \frac{1+\sqrt{\varrho}}{1-\sqrt{\varrho}}\right)^{-1}{ }_{2} F_{1}\left[\begin{array}{c}
a, b \\
a+b
\end{array} ; \varrho\right]=\frac{1}{2},
$$

and

$$
\lim _{\varrho \rightarrow 1^{-}} \frac{\varphi(\varrho)}{\psi(\varrho)}=\lim _{\varrho \rightarrow 1^{-}} \sqrt{\varrho}\left(\log \frac{1+\sqrt{\varrho}}{1-\sqrt{\varrho}}\right)^{-1} \frac{\Gamma(a+b)}{\Gamma(a) \Gamma(b)} \log \frac{1}{1-\varrho}=\frac{\Gamma(a+b)}{\Gamma(a) \Gamma(b)},
$$

where the next to the last equality follows from the formula ([3, Theorem 2.1.3])

$$
\lim _{\varrho \rightarrow 1^{-}}\left(\log \frac{1}{1-\varrho}\right)^{-1}{ }_{2} F_{1}\left[\begin{array}{c}
a, b \\
a+b
\end{array} \varrho\right]=\frac{\Gamma(a+b)}{\Gamma(a) \Gamma(b)} .
$$

The optimality of the constants involved in (2.6) is also proved. 


\section{Proofs of Theorems 1.1 and 1.2}

We begin by recalling two formulas: for $x \in \mathbf{B}_{n}, s \in \mathbf{R}$ and $t>-1$,

$$
\mathbf{I}_{s}(x)={ }_{2} F_{1}\left[\frac{\frac{n-1+s}{2}}{\frac{n}{2}}, \frac{1+s}{2} ;|x|^{2}\right]
$$

and

$$
\mathbf{J}_{s, t}(x)=\frac{\Gamma\left(\frac{n}{2}+1\right) \Gamma(1+t)}{\Gamma\left(\frac{n}{2}+1+t\right)}{ }_{2} F_{1}\left[\begin{array}{c}
\frac{n+t+s}{2}, \frac{2+t+s}{2} \\
\frac{n}{2}+1+t
\end{array}|x|^{2}\right] .
$$

See [9, Lemma 2.1] and [10, Lemma 2.2] for the proofs. (Note that there is a typo in [10, Lemma 2.2]. The exponent in the integrand on the left-hand side of the formula should be $\lambda$ instead of $2 \lambda$.)

In view of (3.2), the assertions (i) and (ii) of Theorems 1.1 and 1.2 follow immediately from Lemma 2.1. The assertions (iii) and (iv) of Theorems 1.1 and 1.2 also follow, since it is easy to check, by using (3.1), (3.2) and (2.3), that

$$
\left(1-|x|^{2}\right)^{s} \mathbf{I}_{s}(x)=\mathbf{I}_{-s}(x) \text { and }\left(1-|x|^{2}\right)^{s} \mathbf{J}_{s, t}(x)=\mathbf{J}_{-s, t}(x) .
$$

To prove (v) and (vi) of Theorems 1.1 and 1.2 we shall apply Lemma 2.2 and Proposition 2.3. We give only the proof for the assertion (vi) of Theorem 1.2; the others are similar and easier. Note that if either $t>-1$ and $n \geq 3$, or $t>0$ and $n \geq 2$, then $(n+t) / 2>0,(2+t) / 2>0$ and

$$
\left(\frac{n+t}{2}\right)\left(\frac{2+t}{2}\right)>\max \left\{\frac{1}{2}, \frac{1}{3}\left(\frac{n+t}{2}+\frac{2+t}{2}\right)\right\} \text {. }
$$

Thus we can combine Proposition 2.3 with (3.2) to get the assertion (vi) of Theorem 1.2 .

\section{Examples}

We present two examples to illustrate the use of our main results, especially, Corollary 1.3. We claim no originality for the results presented in this section.

4.1. Example 1: Norm estimates. If $T$ is a bounded linear operator from $L^{p}\left(\mathbf{B}_{n}\right)$ to $L^{q}\left(\mathbf{B}_{n}\right)$, we write $\|T\|_{p \rightarrow q}$ for the usual operator norm of $T$. Namely,

$$
\|T\|_{p \rightarrow q}:=\sup \left\{\frac{\|T f\|_{q}}{\|f\|_{p}}: f \in L^{p}\left(\mathbf{B}_{n}\right), f \neq 0\right\} .
$$

Suppose $t>-1$. Let $T_{t}$ be the integral operator defined by

$$
T_{t} f(x):=\int_{\mathbf{B}_{n}} K_{t}(x, y) f(y) d V(y),
$$

where

$$
K_{t}(x, y):=\frac{\left(1-|y|^{2}\right)^{t}}{\llbracket x, y \rrbracket^{n+t}} .
$$

The following proposition is slightly less general than [14, Corollary 1.2]. We give a simpler proof here to illustrate the use of Corollary 1.3.

Proposition 4.1. Let $1<p<\infty$ and $p^{\prime}=p /(p-1)$ be the conjugate index of $p$. 
(i) If either $\frac{1}{p}-1<t \leq \frac{2}{p}$ or $t \geq n-2+\frac{2}{p}$, then

$$
\left\|T_{t}\right\|_{p \rightarrow p} \leq \frac{\Gamma\left(\frac{n}{2}+1\right) \Gamma\left(1+t-\frac{1}{p}\right) \Gamma\left(\frac{1}{p}\right)}{\Gamma\left(\frac{2+t}{2}\right) \Gamma\left(\frac{n+t}{2}\right)} ;
$$

(ii) If $\frac{2}{p}<t<n-2+\frac{2}{p}$, then

$$
\left\|T_{t}\right\|_{p \rightarrow p} \leq\left\{\frac{\Gamma\left(\frac{n}{2}+1\right) \Gamma\left(1+t-\frac{1}{p}\right) \Gamma\left(\frac{1}{p}\right)}{\Gamma\left(\frac{2+t}{2}\right) \Gamma\left(\frac{n+t}{2}\right)}\right\}^{\frac{1}{p^{\prime}}}\left\{\frac{\Gamma\left(\frac{n}{2}+1\right) \Gamma\left(\frac{1}{p}\right)}{\Gamma\left(\frac{n}{2}+\frac{1}{p}\right)}\right\}^{\frac{1}{p}} .
$$

Proof. Put

$$
h(x):=\left(1-|x|^{2}\right)^{-\frac{1}{p p^{\prime}}}, \quad x \in \mathbf{B}_{n} .
$$

By Corollary 1.3, we have

$$
\begin{aligned}
& \int_{\mathbf{B}_{n}} K_{t}(x, y) h(y)^{p^{\prime}} d V(y)=\int_{\mathbf{B}_{n}} \frac{\left(1-|y|^{2}\right)^{t-\frac{1}{p}}}{\llbracket x, y \rrbracket^{n+t}} d V(y) \\
& \leq \sup _{x \in \mathbf{B}_{n}}\left\{\int_{\mathbf{B}_{n}} \frac{\left(1-|x|^{2}\right)^{\frac{1}{p}}\left(1-|y|^{2}\right)^{t-\frac{1}{p}}}{\llbracket x, y \rrbracket^{n+t}} d V(y)\right\}\left(1-|x|^{2}\right)^{-\frac{1}{p}}=C_{1} h(x)^{p^{\prime}},
\end{aligned}
$$

for every $x \in \mathbf{B}_{n}$, where

$$
C_{1}:=\frac{\Gamma\left(\frac{n}{2}+1\right) \Gamma\left(1+t-\frac{1}{p}\right) \Gamma\left(\frac{1}{p}\right)}{\Gamma\left(\frac{2+t}{2}\right) \Gamma\left(\frac{n+t}{2}\right)} .
$$

Similarly,

$$
\begin{aligned}
& \int_{\mathbf{B}_{n}} K_{t}(x, y) h(x)^{p} d V(x) \\
& \leq \sup _{y \in \mathbf{B}_{n}}\left\{\int_{\mathbf{B}_{n}} \frac{\left(1-|y|^{2}\right)^{t+\frac{1}{p^{\prime}}}\left(1-|x|^{2}\right)^{-\frac{1}{p^{\prime}}}}{\llbracket x, y \rrbracket^{n+t}} d V(x)\right\}\left(1-|y|^{2}\right)^{-\frac{1}{p^{\prime}}}=C_{2} h(y)^{p}
\end{aligned}
$$

for every $y \in \mathbf{B}_{n}$, where

$$
C_{2}:= \begin{cases}\frac{\Gamma\left(\frac{n}{2}+1\right) \Gamma\left(1+t-\frac{1}{p}\right) \Gamma\left(\frac{1}{p}\right)}{\Gamma\left(\frac{2+t}{2}\right) \Gamma\left(\frac{n+t}{2}\right)}, & \text { if } t \in\left(\frac{1}{p}-1, \frac{2}{p}\right] \cup\left[n-2+\frac{2}{p}, \infty\right) ; \\ \frac{\Gamma\left(\frac{n}{2}+1\right) \Gamma\left(\frac{1}{p}\right)}{\Gamma\left(\frac{n}{2}+\frac{1}{p}\right)}, & \text { if } t \in\left(\frac{2}{p}, n-2+\frac{2}{p}\right) .\end{cases}
$$

The results now follow by an application of Schur's test (see, for example, [16, Theorem 3.8]).

The operators $T_{t}$ are closely related to the harmonic Bergman spaces. Recall that, for $1 \leq p<\infty$, the harmonic Bergman space $b^{p}\left(\mathbf{B}_{n}\right)$ is the set of all harmonic functions $f$ on $\mathbf{B}_{n}$ for which

$$
\|f\|_{p}:=\left(\int_{\mathbf{B}_{n}}|f(x)|^{p} d V(x)\right)^{\frac{1}{p}}<\infty .
$$

It is a closed subspace of $L^{p}\left(\mathbf{B}_{n}\right)$. Let $Q$ denote the orthogonal projection of $L^{2}\left(\mathbf{B}_{n}\right)$ onto $b^{2}\left(\mathbf{B}_{n}\right)$, known as the harmonic Bergman projection. It can be expressed as an integral operator:

$$
Q f(x)=\int_{\mathbf{B}_{n}} f(y) R(x, y) d V(y)
$$


where

$$
R(x, y)=\frac{1}{\llbracket x, y \rrbracket^{n}}\left\{\left(\frac{1-|x|^{2}|y|^{2}}{\llbracket x, y \rrbracket}\right)^{2}-\frac{4|x|^{2}|y|^{2}}{n}\right\} .
$$

It is easy to verify that $|R(x, y)| \leq 4 K_{0}(x, y)$ for all $x, y \in \mathbf{B}_{n}$, and hence $|Q f| \leq$ $4 T_{0}|f|$. Thus Proposition 4.1 implies the following.

Proposition 4.2. [13, Theorem 3.1] If $1<p<\infty$ then $Q$ maps $L^{p}\left(\mathbf{B}_{n}\right)$ boundedly onto $b^{p}\left(\mathbf{B}_{n}\right)$. Moreover, we have $\|Q\|_{p \rightarrow p} \leq 2 n p^{2} /(p-1)$ for all $1<p<\infty$.

4.2. Example 2: An inequality of Hardy-Littlewood type. Recall that the Poisson integral $P[f]$ of a function $f \in L^{1}\left(\mathbf{S}^{n-1}\right)$ is defined by

$$
P[f](x)=\int_{\mathbf{S}^{n-1}} P(x, \zeta) f(\zeta) d \sigma(\zeta), \quad x \in \mathbf{B}_{n},
$$

where $P(x, \zeta)$ denotes the Poisson kernel

$$
P(x, \zeta)=\frac{1-|x|^{2}}{|x-\zeta|^{n}}, \quad x \in \mathbf{B}_{n}, \zeta \in \mathbf{S}^{n-1} .
$$

As usual, for a measurable function $f$ on $\mathbf{B}_{n}$, the means $M_{q}(r, f)$ are defined by

$$
M_{q}(r, f):=\left\{\int_{\mathbf{S}^{n-1}}|f(r \zeta)|^{q} d \sigma(\zeta)\right\}^{\frac{1}{q}}, \quad 0<q<\infty .
$$

The following result is probably well-known, but we have been unable to locate an appropriate reference. It is in the spirit of the classical Hardy-Littlewood inequality concerning the rate of growth of mean values of analytic functions (see [6, Theorem 2]). Also, an $\mathcal{M}$-harmonic analogue can be found in [15, Corollary 4.45].

Proposition 4.3. If $1<p \leq q<\infty$, then there exists a positive constant $C$ such that

$$
M_{q}(r, P[f]) \leq C\left(1-r^{2}\right)^{(1-n)\left(\frac{1}{p}-\frac{1}{q}\right)}\|f\|_{L^{p}\left(\mathbf{S}^{n-1}\right)}
$$

for all $f \in L^{p}\left(\mathbf{S}^{n-1}\right)$ and $0<r<1$.

Corollary 1.3 allows us to give an explicit constant in the above inequality.

Proposition 4.4. One can take the constant $C$ in (4.1) to be

$$
C_{p, q}:= \begin{cases}1, & \text { if } 1 \leq t<\frac{2 n-2}{n} \\ \left\{\frac{\Gamma\left(\frac{n}{2}\right) \Gamma(n t-n+1)}{\Gamma\left(\frac{n t-n+2}{2}\right) \Gamma\left(\frac{n t}{2}\right)}\right\}^{1 / t}, & \text { if } t \geq \frac{2 n-2}{n},\end{cases}
$$

with $t=\frac{p q}{p q+p-q}$.

Proof. Note that $C_{p, p}=1$ for all $p>1$, and in the case when $p=q,(4.1)$ is well-known, see, for example, [4, Theorem 6.4]. So we assume $1<p<q<\infty$ in what follows.

Note first that, by (1.4),

$$
\int_{\mathbf{S}^{n-1}}|P(x, \zeta)|^{t} d \sigma(\zeta) \leq\left(C_{p, q}\right)^{t}\left(1-|x|^{2}\right)^{(1-n)(t-1)}
$$

with the constant $C_{p, q}$ defined as in (4.2).

Now we let $a=q, b=t /(t-1)$ and $c=p /(p-1)$. Then

$$
\frac{1}{a}+\frac{1}{b}=\frac{1}{p}, \quad \frac{1}{a}+\frac{1}{c}=\frac{1}{t}, \quad \frac{1}{a}+\frac{1}{b}+\frac{1}{c}=1 .
$$


Hence, by Hölder's inequality, we obtain

$$
\begin{aligned}
|P f(x)| \leq & \int_{\mathbf{S}^{n-1}}|f(\zeta)||P(x, \zeta)| d \sigma(\zeta) \\
= & \int_{\mathbf{S}^{n-1}}\left\{|P(x, \zeta)|^{\frac{t}{a}}|f(\zeta)|^{\frac{p}{a}}\right\}\left\{|f(\zeta)|^{\frac{p}{b}}\right\}\left\{|P(x, \zeta)|^{\frac{t}{c}}\right\} d \sigma(\zeta) \\
\leq & \left\{\int_{\mathbf{S}^{n-1}}|P(x, \zeta)|^{t}|f(\zeta)|^{p} d \sigma(\zeta)\right\}^{\frac{1}{a}}\left\{\int_{\mathbf{S}^{n-1}}|f(\zeta)|^{p} d \sigma(\zeta)\right\}^{\frac{1}{b}} \\
& \times\left\{\int_{\mathbf{S}^{n-1}}|P(x, \zeta)|^{t} d \sigma(\zeta)\right\}^{\frac{1}{c}} .
\end{aligned}
$$

Combined with (4.3), this yields

$$
|P f(x)|^{q} \leq\left(C_{p, q}\right)^{\frac{t q}{c}}\left(1-|x|^{2}\right)^{\frac{(1-n)(t-1) q}{c}}\|f\|_{L^{p}\left(\mathbf{S}^{n-1}\right)}^{\frac{p q}{b}} \int_{\mathbf{S}^{n-1}}|P(x, \zeta)|^{t}|f(\zeta)|^{p} d \sigma(\zeta)
$$

Let $x=r \eta$, integrate with respect to $\eta$, and apply Fubini's theorem. We get

$$
\int_{\mathbf{S}^{n-1}}|P f(r \eta)|^{q} d \sigma(\eta) \leq\left(C_{p, q}\right)^{q}\left(1-r^{2}\right)^{(1-n)(t-1)\left(\frac{q}{c}+1\right)}\|f\|_{L^{p}\left(\mathbf{S}^{n-1}\right)}^{\frac{p q}{b}+p} .
$$

This proves the desired inequality.

Acknowledgement. Part of this work was done while the second-named author was visiting the Department of Mathematics and Statistics, University of Helsinki. He wishes to express his gratitude for the warm hospitality he received there, especially from Professors Mats Gyllenberg, Pertti Mattila, Tuomas P. Hytönen, Hans-Olav Tylli and Jari Taskinen.

\section{References}

[1] Anderson, G. D., R. W. Barnard, K. C. Richards, M. K. Vamanamurthy, and M. Vuorinen: Inequalities for zero-balanced hypergeometric functions. - Trans. Amer. Math. Soc. 347, 1995, 1713-1723.

[2] Anderson, G. D., M. K. Vamanamurthy, and M. Vuorinen: Inequalities for quasiconformal mappings in space. - Pacific J. Math. 160, 1993, 1-18.

[3] Andrews, G., R. Askey, and R. Roy: Special functions. - Cambridge Univ. Press, Cambridge, 1999.

[4] Axler, S., P. Bourdon, and W. Ramey: Harmonic function theory. 2nd edition. - Grad. Texts in Math. 137, Springer-Verlag, New York, 2001.

[5] Choe, B. R., and H. Koo: Derivatives of harmonic Bergman and Bloch functions on the ball. - J. Math. Anal. Appl. 260, 2001, 100-123.

[6] Hardy, G. H., and J. E. Littlewood: A convergence criterion for Fourier series. - Math. Z. 28, 1928, 612-634.

[7] Jevtić, M., and M. Pavlović: Harmonic Bergman functions on the unit ball in $\mathbf{R}^{n}$. - Acta Math. Hungr. 85, 1999, 81-96.

[8] LiU, C.: Sharp Forelli-Rudin estimates and the norm of the Bergman projection. - J. Funct. Anal. 268, 2015, 255-277.

[9] Liu, C., and L. Peng: Boundary regularity in the Dirichlet problem for the invariant Laplacian $\Delta_{\gamma}$ on the unit real ball. - Proc. Amer. Math. Soc. 132:11, 2004, 3259-3268.

[10] LiU, C., and L. Zhou: Norm of an integral operator related to the harmonic Bergman projection. - Integral Equations Operator Theory 69, 2011, 557-566. 
[11] Ren, G.: Harmonic Bergman spaces with small exponents in the unit ball. - Collect. Math. $53,2002,83-98$.

[12] Rudin, W.: Fuction theory in the unit ball of $\mathbf{C}^{n}$. - Springer-Verlag, New York, 1980.

[13] Stroethoff, K.: Harmonic Bergman spaces. - In: Holomorphic Spaces, MSRI Publications, Volume 33, 1998, 51-63.

[14] Zhou, L.: On the boundedness and the norm of a class of integral operators. - Acta Math. Sci. Ser. B Engl. Ed. 35:6, 2015, 1-8.

[15] ZHU, K.: Spaces of holomorphic functions in the unit ball. - Grad. Texts in Math. 226, Springer, New York, 2005.

[16] ZHU, K.: Operator theory in function spaces. 2nd edition. - Math. Surveys Monogr. 138, Amer. Math. Soc., Providence, 2007.

Received 26 November $2014 \bullet$ Accepted 25 August 2015 